DOI 10. 18307/2019. 0517

(c) 2019 by Journal of Lake Sciences

\title{
针改性膨润土对浅水湖泊水体磷浓度和沉积物磷形态的影响"
}

\author{
甘 磊 $^{1}$, 钟 萍 $^{1}$, 苏 玲 $^{1}$, 刘正文 ${ }^{1,2 * *}$ \\ (1:暨南大学生态学系与水生生物研究中心,广州 510632) \\ (2: 中国科学院南京地理与湖泊研究所, 南京 210008)
}

\begin{abstract}
摘 要: 分析湖泊沉积物磷形态的变化是揭示控制沉积物磷释放机理的关键. 本研究分析了暨南大学南湖加镧改性膨润 土前后水体磷浓度和沉积物磷形态变化, 以探讨镧改性膨润土对削减水体磷浓度的效果. 结果显示, 镧改性膨润土添加 后, 上覆水磷浓度波动较大, 总体呈下降趋势; 至 $60 \mathrm{~d}$ 时, 水体总磷、颗粒态磷、总溶解磷和溶解性反应磷分别比添加前下 降了 $45.1 \% 、 44.1 \%$ 、 $48.2 \%$ 和 $85.3 \%$; 沉积物中的弱结合态磷和氧化还原敏感性磷含量减少, 更稳定的磷灰石结合态磷和 残渣磷含量增加, 而金属氧化物磷和有机磷含量变化较小. 本研究表明针改性膨润土能改变沉积物磷形态, 使活性磷转 化为稳定的磷形态, 从而提高沉积物对磷的滞留能力、降低湖水磷含量, 改善水质.
\end{abstract}

关键词: 锞改性膨润土; 浅水湖泊;沉积物磷形态; 水质

\section{Effects of Lanthanum modified bentonite on the water phosphorus concentration and sedi- ment phosphorus form in a shallow eutrophic lake}

GAN Lei ${ }^{1}$, ZHONG Ping ${ }^{1}$, SU Ling ${ }^{1} \&$ LIU Zhengwen ${ }^{1,2 * *}$

(1: Department of Ecology and Institute of Hydrobiology, Jinan University, Guangzhou 510632, P.R. China)

(2: Nanjing Institute of Geography and Limnology, Chinese Academy of Sciences, Nanjing 210008, P.R.China)

\begin{abstract}
Analyzing the changes in phosphorus ( P ) forms of sediments is the key to understanding the mechanisms of sediment $\mathrm{P}$ release control. This study investigated the changes in water $\mathrm{P}$ concentrations and sediment $\mathrm{P}$-fractions before and after the application of Lanthanum modified bentonite in South Lake in Jinan University in order to analyze the effect on water P concentrations. The results showed that the total $\mathrm{P}$ concentration of the lake water decreased by $45.1 \%$ after 60 days of water $\mathrm{P}$ concentration of the lake changed dramatically after the treatment, after 60 days of the addition of Lanthanum modified bentonite. Meanwhile, the contents of labile-P and reductant-soluble-P were reduced and that of more stable apatite-P and residual-P increased after the treatment, but metal-oxide-P and organic-P did not change. These findings indicate that lanthanum modified bentonite can change sediment $\mathrm{P}$ forms and reduce the contents of the potentially releasable sediment $\mathrm{P}$ forms which likely contribute to the reduced $\mathrm{P}$ concentration in the lake water and thus improve water quality.
\end{abstract}

Keywords: Lanthanum modified bentonite; shallow lakes; sediment phosphorus forms; water quality

水体富营养化是全球最主要的水环境问题 ${ }^{[1]}$. 减少营养盐的输人是解决富营养化问题的关键. 研究表 明, 磷是水体富营养化的主要限制因子, 磷负荷的削减成为富营养化治理的关键 ${ }^{[2]}$. 然而, 在外源磷负荷得 到控制后, 湖泊的水质好转仍需多年, 甚至数十年之久 ${ }^{[3]}$. 湖泊水质对外源污染负荷削减响应延迟的主要原 因之一是沉积物中积累了大量的磷, 会向上覆水体再释放, 形成内源磷负荷, 较长时间地维持水中磷的高浓 度 ${ }^{[4]}$. 因此, 在削减外源磷输人后, 控制湖泊内源磷负荷是加快湖泊修复的关键治理措施 ${ }^{[3]}$.

近年来, 针改性膨润土应用于控制沉积物磷释放受到了关注 ${ }^{[6]}$, 其主要成分是稀土镧和膨润土, 结合在 膨润土中的稀土镧能与溶解 $\mathrm{PO}_{4}^{3-}$ 发生化学反应形成稳定的磷锞镨矿等难溶化合物 $\left(\mathrm{LaPO}_{4} \cdot \mathrm{nH}_{2} \mathrm{O}\right)^{[7-8]}$. 镧

* 国家自然科学基金项目 (41471086)资助. 2018-11-15 收稿;2019-04-01 收修改稿. 甘否( 1991 ), 男, 硕士研究 生;E-mail:ganleigaoan@163.com.

** 通信作者;E-mail:zliu@ niglas.ac.cn. 
改性膨润土不仅能吸附上覆水体中的磷, 其沉降至沉积物表面形成的覆盖层, 还能增强沉积物对磷的滞留 能力, 降低沉积物磷的释放速率 ${ }^{[9-10]}$; 在厌氧条件下 ${ }^{[11-12]}$ 以及 $\mathrm{pH}$ 为 $5 \sim 9$ 的范围内 ${ }^{[11]}$, 镧改性膨润土固定的 磷能稳定地保留在沉积物中. 镧改性膨润土在全球约 200 处水体中得以应用 ${ }^{[6,8]}$, 在欧洲、澳洲和北美洲等 一些富营养化水体磷控制工程中取得了比较理想的效果 ${ }^{[12-13]}$. 虽然国内也开展了一些关于镧改性膨润土的 应用研究, 但多为围隔或实验室研究, 而全湖实验还很少见, 全湖实验能更全面地分析针改性膨润土的效果.

本研究分析了暨南大学南湖加镧改性膨润土前后水体磷浓度以及沉积物磷形态的变化, 包括弱结合态 磷 $\left(\mathrm{NH}_{4} \mathrm{Cl}-\mathrm{P}\right)$ 、氧化还原敏感性磷 $(\mathrm{BD}-\mathrm{P})$ 、金属氧化物磷 $(\mathrm{NaOH}-\mathrm{RP})$ 、有机磷 $(\mathrm{Org}-\mathrm{P}) 、$ 磷灰石结合态磷 (HCl-P) 和残渣磷 (Ref-P), 以探讨锚改性膨润土对沉积物磷释放的影响, 为镧改性膨润土在浅水湖泊富营 养化治理中的应用提供依据.

\section{1 材料与方法}

\section{1 研究湖泊与钵改性膨润土介绍}

研究在暨南大学南湖 $\left(23^{\circ} 07^{\prime} \mathrm{N}, 113^{\circ} 20^{\prime} \mathrm{E}\right.$ ) 进行, 该湖位于广东省广州市 (图 1), 是一个浅水型景观湖 泊,建于 1958 年, 年平均水深约 $1.0 \mathrm{~m}$, 面积约 $4000 \mathrm{~m}^{2}$, 水体富营养化严重 ${ }^{[14]}$. 为治理湖泊富营养化, 于 2013 年完成了截污工程, 2014 年实施了以沉水植物恢复为主的生态修复, 取得了明显的效果 ${ }^{[14]}$. 然而, 2015 年 2 月出现了截污管道堵塞, 大量污水进入暨南大学南湖, 加上福寿螺的影响, 沉水植物消亡, 湖泊又转变 为典型的富营养化浑水态生态系统.

治理技术有限公司提供. 经测定, 锞改性膨润土含针铝和磷分别为 $4.95 \% 、 7.80 \%$ 和 $132.9 \mu \mathrm{g} / \mathrm{g} .2016$ 年 4 月 $28 、 29$ 日, 完成镧改性膨润土添加实验, 添加镧改性膨润土的质量与沉积物表层 $5 \mathrm{~cm}$ 中 $\mathrm{P}_{\text {mobile }}\left(\mathrm{NH}_{4}\right.$ Cl-P、BD-P 和 Org-P 之和) 以及上覆水体 TP 之和的质量比为 $100: 1$, 添加量见表 1. 镧改性膨润土以泥浆的 形式均匀喷酒于湖面.

表 1 锞改性膨润土添加量*

Tab. 1 The dose calculation of the Lanthanum modified bentonite

\begin{tabular}{|c|c|c|c|c|c|c|c|}
\hline \multicolumn{3}{|c|}{ 沉积物 } & \multicolumn{2}{|c|}{ 上覆水 } & \multirow{2}{*}{$\begin{array}{c}\text { 内源活性磷 } \\
\text { 总量/kg }\end{array}$} & \multirow{2}{*}{$\begin{array}{l}\text { 添加 } \\
\text { 质量比 }\end{array}$} & \multirow{2}{*}{$\begin{array}{l}\text { 针改性膨润 } \\
\text { 土添加量/ } \mathrm{t}\end{array}$} \\
\hline $\begin{array}{c}\text { 活性磷含量/ } \\
(\mu \mathrm{g} / \mathrm{g})\end{array}$ & $\begin{array}{l}\text { 平均密度/ } \\
\left(\mathrm{g} / \mathrm{cm}^{3}\right)\end{array}$ & $\begin{array}{c}\text { 活性磷总量/ } \\
\mathrm{kg}\end{array}$ & $\begin{array}{c}\text { 总磷浓度/ } \\
(\mathrm{mg} / \mathrm{L})\end{array}$ & $\begin{array}{c}\text { 总磷总量/ } \\
\text { kg }\end{array}$ & & & \\
\hline 76.25 & 1.50 & 22.89 & 0.16 & 0.72 & 23.61 & $100: 1$ & 2.36 \\
\hline
\end{tabular}

* 沉积物活性磷依据表层 $5 \mathrm{~cm}$ 计算, 水体面积为 $4000 \mathrm{~m}^{2}$, 水深按 $1 \mathrm{~m}$ 计算.

\section{2 水样采集与分析方法}

上覆水水质背景值采样时间为 2015 年 6 月- 2016 年 4 月,每两周采集 1 次,所有水质数据取平均值. 试验期间采集水样的时间为 2016 年 4 月 26 日 -6 月 27 日,采样频率前半个月为每天 1 次,第 2 个半月为 3 天 1 次, 1 个月后为每周 1 次. 采样点如图 1 所示, 每次的采样时间均为上午 $8: 00$ 点, 用 $5 \mathrm{~L}$ 有机玻璃采水器 采集水面表层以下约 $50 \mathrm{~cm}$ 的水样,低温保存,带回实验室进行常规水质指标的测定.

上覆水体中总磷 (TP) 、总溶解磷 (TDP) 和溶解性反应磷 ( SRP) 浓度的检测采用钿锑抗分光光度法, 颗 粒态磷 $(\mathrm{PP})$ 浓度为 TP 浓度与 TDP 浓度之差. 总氮 (TN) 浓度的测定采用碱性过硫酸钾紫外分光光度法, 浮游植物叶绿素 a (Chl.a) 浓度的测定采用丙酮萃取分光光度法 ${ }^{[16]}$. 现场用赛氏透明度盘测定水体透明度 $(\mathrm{SD})$, 用 YSI-556 多参数水质分析测试仪现场测定水温 ( T)、溶解氧 ( DO ) 、 $\mathrm{pH}$ 等.

\section{3 沉积物样品采集与分析方法}

分别于 2016 年 3 月 22 日（背景值）、2016 年 4 月 26 日（加镧改性膨润土前 $2 \mathrm{~d}$ ) 和 2016 年 6 月 28 日 (加镧改性膨润土后 $60 \mathrm{~d}$ ) 采集沉积物样品, 采样点与水样采样点相同 (图 1). 每个位点采集 2 个, 共 6 个 平行样品. 用柱状采泥器 (有机玻璃管长 $32 \mathrm{~cm}$, 内径 $8.5 \mathrm{~cm}$ ) 来采集, 采集沉积物深度约 $20 \mathrm{~cm}$. 样品现场 垂直切割, $0 \sim 10 \mathrm{~cm}$ 的样品间隔 $2 \mathrm{~cm}$ 切割, 10 15 $\mathrm{cm}$ 作为一个分层样品进行处理. 将切割后的样品装人聚 
乙烯封口袋内, 排尽袋内空气密封、编号,带回实验室保存于 $4^{\circ} \mathrm{C}$ 冰箱中待分析.

沉积物磷提取方法参考文献 ${ }^{[17-18]}$, 具体步骤如下: (1) $1 \mathrm{~g}$ 沉积物新鲜样品, $1 \mathrm{~mol} / \mathrm{L} \mathrm{NH}_{4} \mathrm{Cl}$ 溶液并调节 $\mathrm{pH}=7,25^{\circ} \mathrm{C}$ 条件下震荡 $2 \mathrm{~h}, 3500$ 转 $/ \mathrm{min}$, 离心 $10 \mathrm{~min}$, 经 $0.45 \mu \mathrm{m}$ 的醋酸纤维膜过滤后, 提取 $\mathrm{NH}_{4} \mathrm{Cl}-\mathrm{P}$; (2) 步骤 (1) 剩余的残渣, $0.11 \mathrm{~mol} / \mathrm{L} \mathrm{NaHCO}$ 溶液和 $0.11 \mathrm{~mol} / \mathrm{L} \mathrm{Na}_{2} \mathrm{~S}_{2} \mathrm{O}_{4}$ 溶液 ( $\mathrm{BD}$ 溶液), $40^{\circ} \mathrm{C}$ 条件下震荡 $1 \mathrm{~h}$, 3500 转 $/ \mathrm{min}$, 离心 $10 \mathrm{~min}$, 经 $0.45 \mu \mathrm{m}$ 的醋酸纤维膜过滤后, 提取 BD-P; ( 3a) 步骤 (2) 剩余的残渣, 0.1 $\mathrm{mol} / \mathrm{L} \mathrm{NaOH}$ 溶液, $25^{\circ} \mathrm{C}$ 条件下震荡 $16 \mathrm{~h}, 3500$ 转 $/ \mathrm{min}$, 离心 $10 \mathrm{~min}$, 经 $0.45 \mu \mathrm{m}$ 的醋酸纤维膜过滤后, 提取 $\mathrm{NaOH}-\mathrm{RP}$; (3b) 3a 步骤中的提取液用过硫酸钾溶液消解后测定其总磷 ( NaOH-TP) 含量, 此值与 NaOH-RP 的差值为 NaOH-NRP, 即有机磷 (Org-P) ; (4) 步骤 (3a) 剩余的残渣, $0.5 \mathrm{~mol} / \mathrm{L} \mathrm{HCl}$ 溶液 $25^{\circ} \mathrm{C}$ 震荡 $16 \mathrm{~h}$, 3500 转 $/ \mathrm{min}$, 离心 $10 \mathrm{~min}$, 经 $0.45 \mu \mathrm{m}$ 的醋酸纤维膜过滤后, 提取 HCl-P; (5) 步骤 (4) 剩余的残渣, $1 \mathrm{~mol} / \mathrm{L}$ $\mathrm{NaOH}$ 溶液, $85^{\circ} \mathrm{C}$ 条件下震荡 $24 \mathrm{~h}, 3500$ 转 $/ \mathrm{min}$, 离心 $10 \mathrm{~min}$, 经 $0.45 \mu \mathrm{m}$ 的醋酸纤维膜过滤后, 提取 Ref-P. 沉积物总磷 (Total-P) 含量为各形态磷含量之和 (沉积物新鲜样品), 沉积物含水率和烧失量分别为 $105^{\circ} \mathrm{C}$ 烘干和 $550^{\circ} \mathrm{C}$ 灼烧后的质量损失, 密度采用质量体积法. $\mathrm{TN}$ 含量通过将风干研磨的泥样加人 $50 \mathrm{ml}$ 去离子 水中混合, 然后加碱性过硫酸钾氧化消解, 采用紫外分光光度法测定.

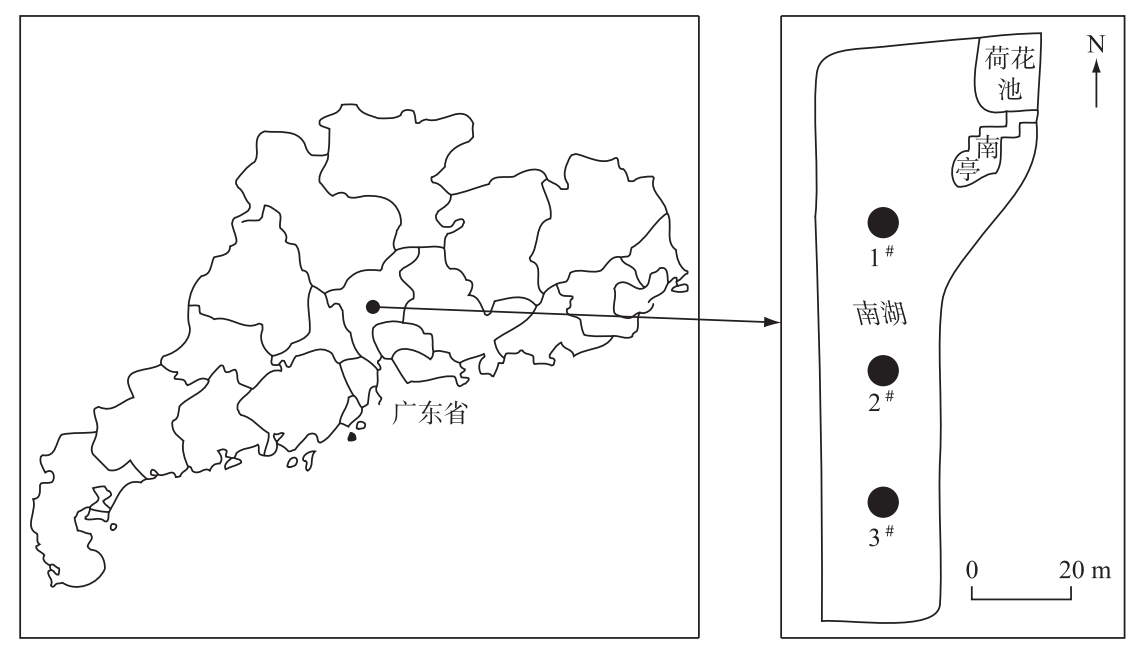

图 1 南湖采样点分布

Fig.1 Distribution of sampling sites in South Lake

\section{4 数据分析及处理}

实验所得数据采用 Excel 2007 软件整理, 采用 SPSS 22.0 统计软件进行方差分析, 加镧改性膨润土前后 的沉积物 TP 及磷形态之间差异性采用两独立样本 $t$ 检验进行分析, 采用 Origin 8.5 软件作图.

\section{2 结果与分析}

\section{1 上覆水体及沉积物性状}

暨南大学南湖 2015 年 6 月 -2016 年 4 月的平均水质状况如表 2 所示. 此期间平均水温为 $23.23^{\circ} \mathrm{C}$, 不存 在人为造成的水温变化, $\mathrm{pH}$ 呈弱碱性, $\mathrm{DO}$ 浓度属于 III 类水质. TN 浓度属于 III 类水质, $\mathrm{TP}$ 浓度属于 IV 类 水质.

暨南大学南湖表层 $15 \mathrm{~cm}$ 沉积物的含水率、密度、烧失量、TN 及各形态磷含量如表 3 所示. 沉积物含水 率在0 15 $\mathrm{cm}$ 深度范围内随深度增加逐渐降低. 表层 $0 \sim 2 \mathrm{~cm}$ 含水率达到 $85.8 \%, 10 \sim 15 \mathrm{~cm}$ 处的含水率下 降至 55.7\%, 比表层 0 2 cm 下降了 $35.1 \%$. 烧失量也呈现出由表层到深层逐渐降低的趋势, 表层 0 2 cm 烧 失量为 $182.0 \mu \mathrm{g} / \mathrm{g}, 10 \sim 15 \mathrm{~cm}$ 处的烧失量为 $129.4 \mu \mathrm{g} / \mathrm{g}$, 比表层 $0 \sim 2 \mathrm{~cm}$ 降低了 $28.9 \%$. 密度随深度增加逐 渐增加, 但增加的幅度不大, 表层密度略低于深层. 表层 $0 \sim 2 \mathrm{~cm}$ 密度最低为 $1.5 \mathrm{~g} / \mathrm{cm}^{3}, 10 \sim 15 \mathrm{~cm}$ 处的密度 
最大为 $1.7 \mathrm{~g} / \mathrm{cm}^{3}$. 沉积物 TN 也呈现出由表层到深层逐渐降低的趋势, 表层 $0 \sim 15 \mathrm{~cm} \mathrm{TN}$ 含量在 2934.4 $5645.4 \mu \mathrm{g} / \mathrm{g}$ 范围内波动, 平均值为 $4577.4 \mu \mathrm{g} / \mathrm{g}, 0 \sim 8 \mathrm{~cm}$ 范围内 TN 含量都在 $5000 \mu \mathrm{g} / \mathrm{g}$ 以上. 沉积物 TP 含 量随深度增加而增加, 因为沉积物磷形态测定的是沉积物新鲜样品, 通过含水率换算, 表层 $0 \sim 15 \mathrm{~cm}$ TP 含 量在 $1109.4 \sim 1629.6 \mu \mathrm{g} / \mathrm{g}$ 范围内波动, 平均值为 $1451.7 \mu \mathrm{g} / \mathrm{g}$. $\mathrm{NH}_{4} \mathrm{Cl}-\mathrm{P}$ 含量在表层 $0 \sim 15 \mathrm{~cm}$ 范围内均较 低, 其中表层 $0 \sim 2 \mathrm{~cm}$ 含量最高, 为 $1.1 \mu \mathrm{g} / \mathrm{g}$. 沉积物中 BD-P 含量在 $0 \sim 15 \mathrm{~cm}$ 范围内波动较大, 平均值为 $26.0 \mu \mathrm{g} / \mathrm{g}$, 其中表层 $0 \sim 2 \mathrm{~cm}$ 含量最高, 达到了 $33.3 \mu \mathrm{g} / \mathrm{g}$, 整体呈现出随深度增加而下降的趋势. Org-P 含量 随沉积物深度增加呈现先升高后降低再升高的趋势, 平均值为 $56.45 \mu \mathrm{g} / \mathrm{g}$, 整体上表层 Org-P 含量低于深 层. NaOH-RP 含量在 $0 \sim 15 \mathrm{~cm}$ 范围内均较高, 其中 $2 \sim 4 \mathrm{~cm}$ 处最低, 为 $91.2 \mu \mathrm{g} / \mathrm{g}, 10 \sim 15 \mathrm{~cm}$ 处最高, 达 $354.5 \mu \mathrm{g} / \mathrm{g}$, 平均值为 $229.9 \mu \mathrm{g} / \mathrm{g}$, 整体上呈现出由沉积物表层到深层逐渐升高的趋势. HCl-P 和 Ref-P 含量 整体上也均呈现出由沉积物表层到深层逐渐升高的趋势, 其中 HCl-P 含量最低值 $(19.2 \mu \mathrm{g} / \mathrm{g})$ 出现在 $0 \sim 2$ $\mathrm{cm}$ 层, 最高值 $(76.6 \mu \mathrm{g} / \mathrm{g}$ ) 出现在 $10 \sim 15 \mathrm{~cm}$ 层, Ref-P 含量最低值 $(19.3 \mu \mathrm{g} / \mathrm{g})$ 出现在 $0 \sim 2 \mathrm{~cm}$ 层, 最高值 $(63.1 \mu \mathrm{g} / \mathrm{g})$ 出现在 $8 \sim 10 \mathrm{~cm}$ 层.

表 2 南湖上覆水体理化性质

Tab.2 The chemical properties of overlying water in South Lake

\begin{tabular}{lccccccccc}
\hline $\begin{array}{c}\text { 温度/ } \\
{ }^{\circ} \mathrm{C}\end{array}$ & $\mathrm{pH}$ & $\begin{array}{c}\mathrm{DO} / \\
(\mathrm{mg} / \mathrm{L})\end{array}$ & $\begin{array}{c}\text { 透明度/ } \\
\mathrm{cm}\end{array}$ & $\begin{array}{c}\mathrm{TN} / \\
(\mathrm{mg} / \mathrm{L})\end{array}$ & $\begin{array}{c}\mathrm{TP} / \\
(\mathrm{mg} / \mathrm{L})\end{array}$ & $\begin{array}{c}\mathrm{PP} / \\
(\mathrm{mg} / \mathrm{L})\end{array}$ & $\begin{array}{c}\mathrm{TDP} / \\
(\mathrm{mg} / \mathrm{L})\end{array}$ & $\begin{array}{c}\mathrm{SRP} / \\
(\mathrm{mg} / \mathrm{L})\end{array}$ & $\begin{array}{c}\mathrm{Chl} . \mathrm{a} / \\
(\mu \mathrm{g} / \mathrm{L})\end{array}$ \\
\hline 23.23 & 7.45 & 5.97 & 70.17 & 1.000 & 0.107 & 0.079 & 0.027 & 0.016 & 53.5 \\
\hline
\end{tabular}

表 3 南湖沉积物性状及各形态磷含量

Tab.3 The sediments character and phosphorus form in South Lake

\begin{tabular}{cccccccccccc}
\hline $\begin{array}{c}\text { 深度/ } \\
\mathrm{cm}\end{array}$ & $\begin{array}{c}\text { 含水率/ } \\
\%\end{array}$ & $\begin{array}{c}\text { 密度/ } \\
\left(\mathrm{g} / \mathrm{cm}^{3}\right)\end{array}$ & $\begin{array}{c}\text { 烧失量/ } \\
(\mu \mathrm{g} / \mathrm{g})\end{array}$ & $\begin{array}{c}\mathrm{TN} / \\
(\mu \mathrm{g} / \mathrm{g})\end{array}$ & $\begin{array}{c}\mathrm{TP} / \\
(\mu \mathrm{g} / \mathrm{g})\end{array}$ & $\begin{array}{c}\mathrm{NH}_{4} \mathrm{Cl}-\mathrm{P} / \\
(\mu \mathrm{g} / \mathrm{g})\end{array}$ & $\begin{array}{c}\mathrm{BD}-\mathrm{P} / \\
(\mu \mathrm{g} / \mathrm{g})\end{array}$ & $\begin{array}{c}\mathrm{Org}-\mathrm{P} / \mathrm{NaOH}-\mathrm{RP} / \mathrm{HCl}-\mathrm{P} / \\
(\mu \mathrm{g} / \mathrm{g})\end{array}$ & $\begin{array}{c}\mathrm{Ref}-\mathrm{P} / \\
(\mu \mathrm{g} / \mathrm{g})\end{array}$ & $(\mu \mathrm{g} / \mathrm{g})$ & $(\mu \mathrm{g} / \mathrm{g})$ \\
\hline $0 \sim 2$ & 85.8 & 1.5 & 182.0 & 5645.4 & 230.7 & 1.1 & 33.3 & 47.9 & 109.9 & 19.2 & 19.3 \\
$2 \sim 4$ & 79.2 & 1.5 & 156.3 & 5345.9 & 230.8 & 0.8 & 23.1 & 56.9 & 91.2 & 34.1 & 24.6 \\
$4 \sim 6$ & 75.4 & 1.6 & 132.9 & 5308.6 & 308.9 & 0.8 & 25.4 & 44.2 & 178.3 & 31.7 & 28.4 \\
$6 \sim 8$ & 73.1 & 1.6 & 121.1 & 5002.9 & 483.6 & 0.9 & 29.8 & 59.2 & 322.7 & 42.9 & 28.0 \\
$8 \sim 10$ & 66.1 & 1.6 & 123.2 & 3227.2 & 546.4 & 0.8 & 22.7 & 63.4 & 322.5 & 73.9 & 63.1 \\
$10 \sim 15$ & 55.7 & 1.7 & 129.4 & 2934.4 & 579.7 & 0.7 & 21.7 & 67.0 & 354.5 & 76.6 & 59.0 \\
\hline
\end{tabular}

\section{2 实验期间上覆水水质变化}

加针改性膨润土后 $1 \mathrm{~d}$ 与加镧改性膨润土前 $2 \mathrm{~d}$ 相比, 上覆水 TP 浓度由 $0.087 \mathrm{mg} / \mathrm{L}$ 上升至 $0.116 \mathrm{mg} /$ $\mathrm{L}, \mathrm{PP}$ 浓度由 $0.058 \mathrm{mg} / \mathrm{L}$ 上升至 $0.098 \mathrm{mg} / \mathrm{L}$, TDP 浓度由 $0.029 \mathrm{mg} / \mathrm{L}$ 下降为 $0.018 \mathrm{mg} / \mathrm{L}, \mathrm{SRP}$ 浓度由 0.022 $\mathrm{mg} / \mathrm{L}$ 下降为 $0.005 \mathrm{mg} / \mathrm{L}$. TP、PP、TDP 和 SRP 浓度在前期均波动较大, 总体呈现出下降的趋势. TP、PP 浓度 在 $33 \mathrm{~d}$ 后趋于平稳, 维持在较低的水平, 至 $60 \mathrm{~d}$ 时, TP 浓度为 $0.048 \mathrm{mg} / \mathrm{L}, \mathrm{PP}$ 浓度为 $0.016 \mathrm{mg} / \mathrm{L}$, 与加镧改 性膨润土前 $2 \mathrm{~d}$ 相比, TP 浓度下降了 $45.1 \%, \mathrm{PP}$ 浓度下降了 $44.1 \%$. TDP 浓度在 $60 \mathrm{~d}$ 时为 $0.015 \mathrm{mg} / \mathrm{L}$, 与加 镧改性膨润土前 $2 \mathrm{~d}$ 相比, TDP 浓度下降了 $48.2 \%$. SRP 浓度在 $23 \mathrm{~d}$ 后还存在波动, 但维持在较低的水平, 至 $60 \mathrm{~d}$ 时与加镧改性膨润土前 $2 \mathrm{~d}$ 相比, SRP 浓度下降了约 $85.3 \%$ (图 2).

实验期间, 上覆水的温度变化范围为 $24.7 \sim 29.2^{\circ} \mathrm{C}$, 整体上都呈现出随时间逐渐升高的趋势. 透明度在 $1 \sim 16 \mathrm{~d}$ 内波动较大, $16 \mathrm{~d}$ 后透明度逐渐增加, 33 60 d 内, 透明度约为 $1 \mathrm{~m}$, 清澈见底. TN 浓度在前期先下降 后上升, 总体上呈下降趋势, 至 $60 \mathrm{~d}$ 时, 下降为 $0.56 \mathrm{mg} / \mathrm{L}$. 叶绿素 $\mathrm{a}$ 浓度在前期稍有波动, 加锚改性膨润土 $7 \sim 60 \mathrm{~d}$ 与加之前 $2 \mathrm{~d}$ 相比变化不大 (图 3).

\section{3 沉积物总磷和磷形态的变化}

$\mathrm{TP}$ 含量在沉积物表层 $0 \sim 6 \mathrm{~cm}$ 均有增加, 但未达到显著水平 $(P>0.05)$, 在 $8 \sim 15 \mathrm{~cm}$ 层添加前后变化不 大 $(P>0.05)$ (图 4). 

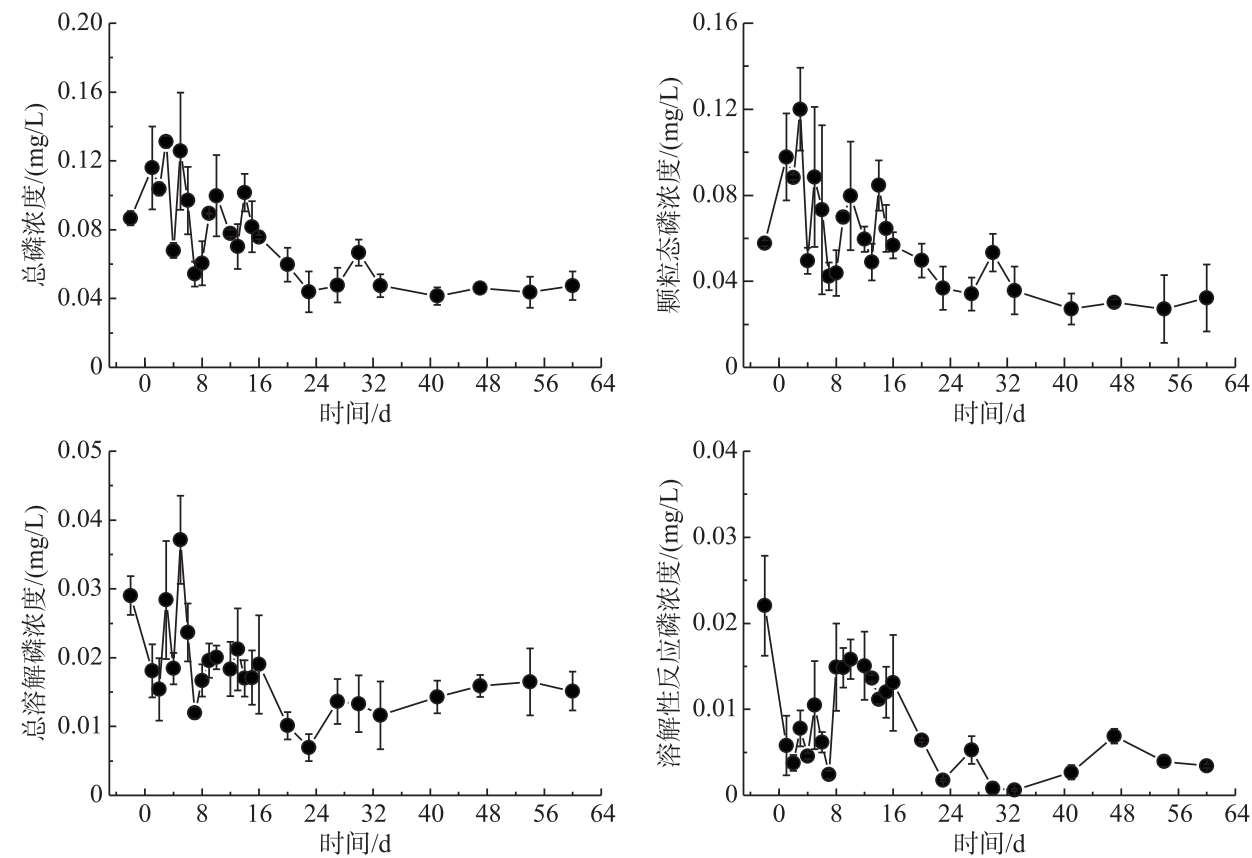

图 2 南湖上覆水中磷浓度变化

Fig.2 Variation of phosphorus concentrations in South Lake water
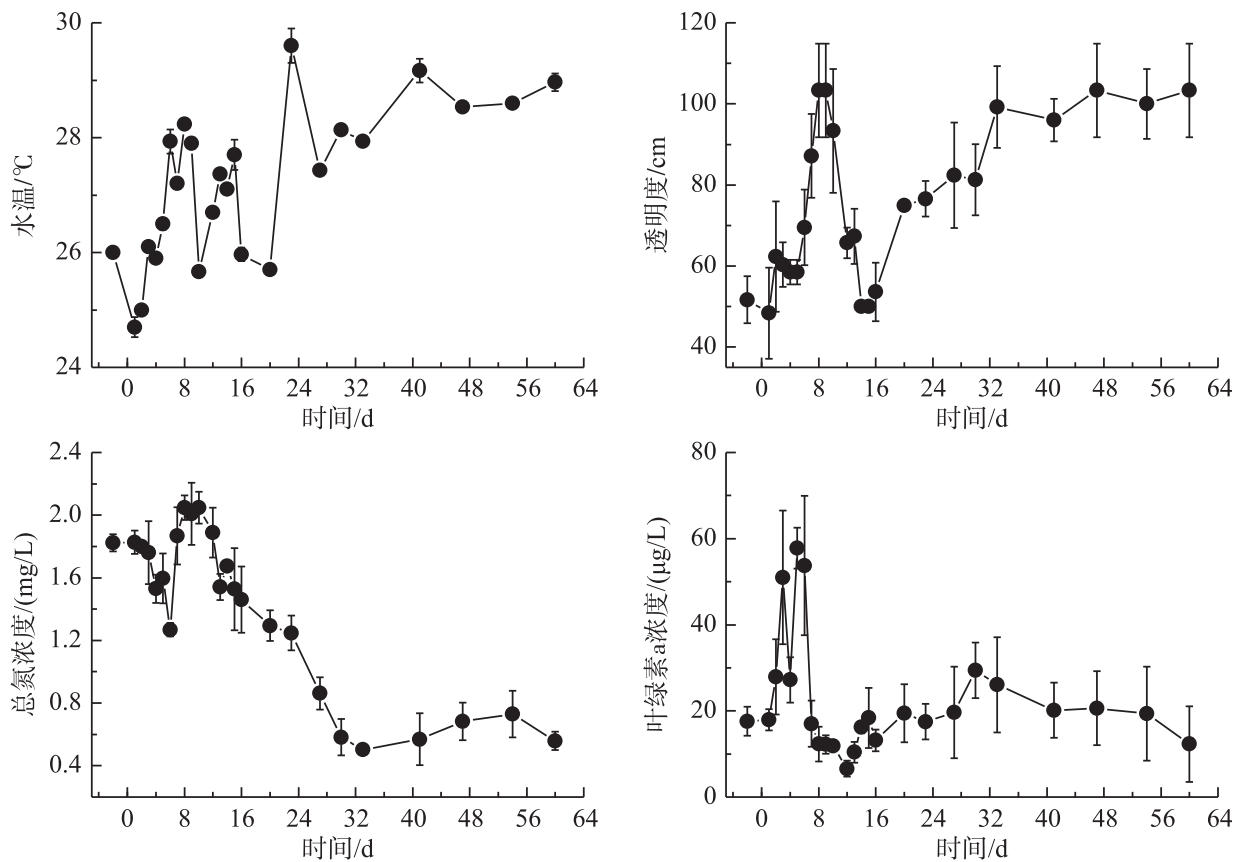

图 3 南湖上覆水水温、透明度、总氮浓度和叶绿素 $\mathrm{a}$ 浓度

Fig. 3 The temperature, transparency, total nitrogen and chlorophyll-a concentrations in South Lake water 


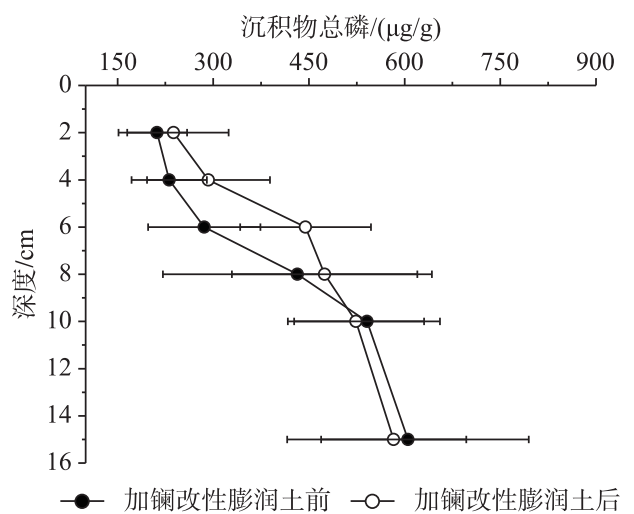

图 4 南湖沉积物总磷垂直分布特征

Fig.4 Vertical distribution of total phosphorus in South Lake sediments
$\mathrm{NH}_{4} \mathrm{Cl}-\mathrm{P}$ 含量在加镧改性膨润土之前占 $\mathrm{TP}$ 的比例为 $0.1 \% \sim 0.4 \%$, 加镧改性膨润土之后下降为 $0.1 \% \sim 0.2 \%$, 同 时除沉积物深度 $4 \sim 6 \mathrm{~cm}$ 外, 其他层均有下降, 特别是表层 $0 \sim 2 \mathrm{~cm}$, 加之后的含量为 $0.50 \mu \mathrm{g} / \mathrm{g}$, 显著低于加之前的 $0.92 \mu \mathrm{g} / \mathrm{g}(P<0.05)$ ( 图 5a). BD-P 含量在加镧改性膨润土 之后, $0 \sim 15 \mathrm{~cm}$ 范围内均显著下降 $(P<0.05)$, 为加镧改性 膨润土之前的 $32.5 \% \sim 56.7 \%$, 其中表层 $0 \sim 2 \mathrm{~cm}$ 下降幅度 最大,下降了 $67.5 \%$ (图 5b). Org-P 和 NaOH-RP 含量在 $0 \sim 15 \mathrm{~cm}$ 层中均无明显变化 $(P>0.05)$ ( 图 5c、d). 加镧改 性膨润土后, $\mathrm{HCl} \sim \mathrm{P}$ 含量在 $0 \sim 6 \mathrm{~cm}$ 范围显著增加 $(P<$ $0.05), 0 \sim 2 \mathrm{~cm}$ 层、2 4 cm 层、 $4 \sim 6 \mathrm{~cm}$ 层分别增加了 $256.7 \% 、 180.7 \% 、 137.6 \%$. 而在 6 15 cm 层变化不明显 $(P>$ 0.05 ) (图 5e). Ref-P 含量在 $0 \sim 8 \mathrm{~cm}$ 层均有增加, 其中 $2 \sim$ $8 \mathrm{~cm}$ 层含量显著高于加镧改性膨润土前 $(P<0.05)$. 在 $8 \sim$ $15 \mathrm{~cm}$ 层变化不明显 $(P>0.05)$ ( 图 5f).

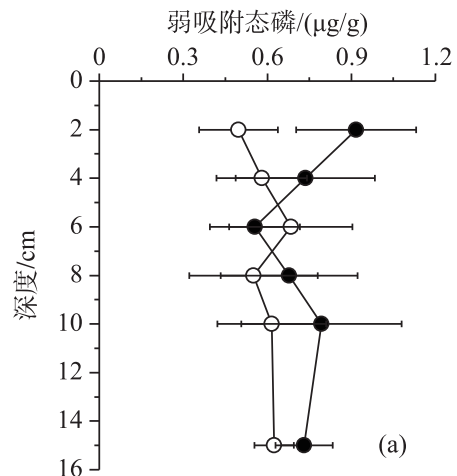

金属氧化物磷 $/(\mu \mathrm{g} / \mathrm{g})$

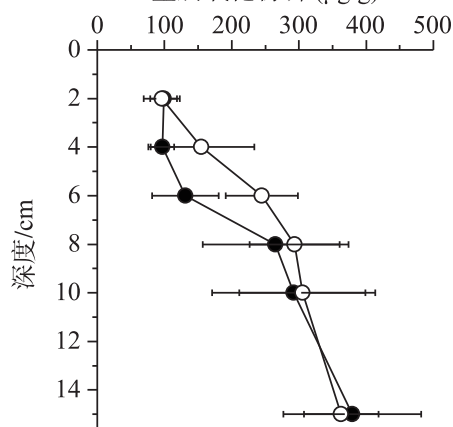

(d)
氧化还原敏感性磷 $/(\mu \mathrm{g} / \mathrm{g})$
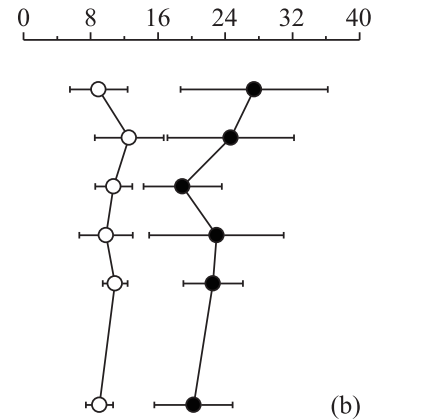

(b)

磷灰石磷 $/(\mu \mathrm{g} / \mathrm{g})$

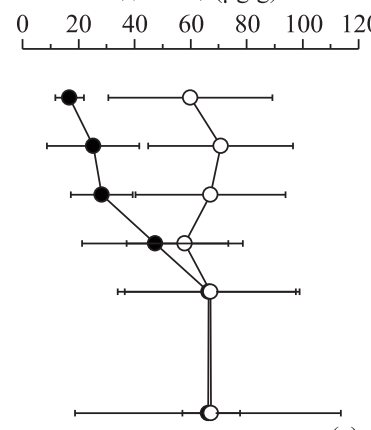

(e)

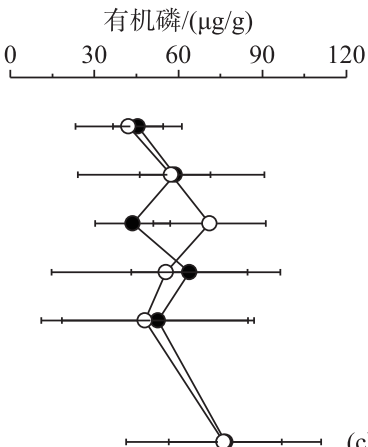

(c)

- - 加针改性膨润土前 - - 加锞改性膨润土后

残渣磷 $/(\mu \mathrm{g} / \mathrm{g})$

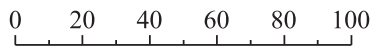

图 5 南湖沉积物磷形态垂直分布特征

Fig.5 Vertical distribution of phosphorus form in South Lake sediments

\section{3 讨论}

\section{1 南湖沉积物性状分析}

含水率高的沉积物往往具有更细的粒径, 质量相对更轻 ${ }^{[19]}$, 含水率的大小直接影响到沉积物再悬浮的 
风险程度, 因而是影响内源磷释放的一个重要因素 ${ }^{[20]}$. 暨南大学南湖沉积物表层 $0 \sim 2 \mathrm{~cm}$ 含水率高达 $85.8 \%$, 表层沉积物具有较大的不稳定性, 容易发生再悬浮. 烧失量的大小与有机质含量密切相关 ${ }^{[21]}$, 南湖 沉积物烧失量含量随沉积物深度增加逐渐降低, 说明表层有机质含量较丰富. 有机质腐烂分解会将部分有 机磷转化为无机磷, 向上覆水释放 ${ }^{[22]}$, 是内源磷的潜在来源.

南湖沉积物 TN 含量平均值为 $4577.4 \mu \mathrm{g} / \mathrm{g}$, 这与杭州西湖 TN 含量最高处西湖底的 TN 含量相当 (平均 值为 $4960 \mu \mathrm{g} / \mathrm{g})^{[23]}$, 远高于太湖 ( TN 含量为 $363.9 \sim 1534.8 \mu \mathrm{g} / \mathrm{g}$ ) ${ }^{[24]}$ 、巢湖 ( TN 平均含量为 $1737.0 \mu \mathrm{g} /$ $\mathrm{g})^{[25]}$ 等典型严重富营养化湖泊. 南湖沉积物 TP 含量平均值为 $1451.7 \mu \mathrm{g} / \mathrm{g}$, 与杭州西湖 TP 含量最高处小 南湖的 TP 含量相当 (平均值为 $1485 \mu \mathrm{g} / \mathrm{g}$ ) ${ }^{[23]}$, 远高于太湖 (平均含量为 $560.4 \mu \mathrm{g} / \mathrm{g}$ ) ${ }^{[26]}$ 和巢湖 (平均含量 为 $691.0 \mu \mathrm{g} / \mathrm{g})^{[25]}$ 的 TP 含量. 说明暨南大学南湖具有非常高的氮、磷内源负荷, 这可能是人类活动所造成的 营养盐的积累, 包括周围生活污水和地表径流的输人, 以及钓鱼者常年饵料的输人等. 2013 年初暨南大学相 关部门对南湖进行了截污处理 ${ }^{[14]}$, 外源污染基本得到了控制, 但内源问题仍是限制南湖水质改善的关键.

$\mathrm{NH}_{4} \mathrm{Cl}-\mathrm{P}$ 是弱吸附态磷, 包括吸附在碳酸盐、铁盐等沉积矿物颗粒表面的磷和间隙水中的可溶性磷, 以 及从植物碎屑中沥滤出来的磷 ${ }^{[18]}$. 有研究表明, 当沉积物环境条件变化或受到扰动时, 该类型的磷最容易 向上覆水体扩散 ${ }^{[27]}$. 南湖表层沉积物中 $\mathrm{NH}_{4} \mathrm{Cl}-\mathrm{P}$ 含量较高, 说明表层沉积物具有较高的释放风险. 南湖沉 积物表层 $0 \sim 15 \mathrm{~cm}$ BD-P 含量平均值为 $26.0 \mu \mathrm{g} / \mathrm{g}$, BD-P 是一种氧化还原敏感性磷, 主要与铁、镇氧化物结 合 ${ }^{[18]}$, 沉积物释放磷的主要来源 ${ }^{[26,28-29]}$. 因此, 南湖的高 BD-P 含量, 可能对内源磷负荷向上覆水体的释放 产生重要的影响. Org-P 多以磷酸酯 (包括肌醇六磷酸)、磷脂、核酸、磷蛋白和磷酸糖类以及一些未知的化 合物等形式存在 ${ }^{[30]}$. 主要来源于生物有机体的沉积, 被认为是潜在可移动磷的重要组成部分. 南湖沉积物 Org-P 含量整体上表层低于深层, 说明 Org-P 发生了一定程度的释放. Org-P 含量平均值为 $56.45 \mu \mathrm{g} / \mathrm{g}$, 是 BD-P 含量的两倍多, Org-P 对上覆水体磷的输人不可忽视.

$\mathrm{NaOH}-\mathrm{RP}$ 是可与氢氧根离子进行交换的铁铝金属氧化物约束的磷, 释放的潜力小于 BD-P 和 $\mathrm{NH}_{4} \mathrm{Cl}-\mathrm{P}$. $\mathrm{NaOH}-\mathrm{RP}$ 被公认为沉积物较为稳定形态磷的组成部分, 许多湖泊修复采用添加铝盐来控制内源磷的释 放 ${ }^{[31-32]}$. 南湖沉积物 NaOH-RP 含量较高, 平均值为 $229.9 \mu \mathrm{g} / \mathrm{g}$, 有利于沉积物对磷的滞留. HCl-P 主要是指 碳酸盐结合的磷和磷灰石磷等, 是一种生物难利用的磷, 相对稳定. Ref-P 是沉积物中最稳定的永久储存的 主要磷形态. HCl-P 和 Ref-P 被认为是沉积物中永久储存的主要磷形态 ${ }^{[18]}$. 尽管 NaOH-RP、HCl-P 和 Ref-P 较稳定, 但影响磷形态转化的因素是多种的, 3 种磷形态均呈现出由沉积物表层到深层逐渐增加的趋势, 说 明还是存在一定程度释放.

沉积物中的 $\mathrm{NH}_{4} \mathrm{Cl}-\mathrm{P} 、 \mathrm{BD}-\mathrm{P} 、 \mathrm{Org}-\mathrm{P}$ 易受环境影响而释放, 被认为是潜在的释放敏感性磷 ${ }^{[12,33-34]}$. 因此本 研究将此 3 种磷作为活性磷 ( $\left.\mathrm{P}_{\text {mobile }}\right)$. 南湖表层 $(0 \sim 15 \mathrm{~cm})$ 沉积物中活性磷占 TP 的 $21.0 \%$. 沉积物中的磷 含量约为上覆水体中磷酸盐含量的数百倍甚至上千倍, 若南湖沉积物中一小部分活性磷发生释放, 对南湖 水体磷含量将产生重要的影响. 因此, 南湖沉积物磷释放的控制还是很必要的.

\section{2 南湖应用镧改性膨润土的效果分析}

镧改性膨润土的主要成分是稀土锞和膨润土, 钵只存在唯一的氧化价态 ( 3 价), $\mathrm{La}^{3+}$ 和 $\mathrm{PO}_{4}^{3-}$ 反应的化 学计量摩尔比是 $1: 1^{[6]}$. Dithmer 等 ${ }^{[35]}$ 利用固态 ${ }^{31} \mathrm{P}$ 核磁共振研究表明, $\mathrm{La}$ 与 $\mathrm{PO}_{4}^{3-}$ 首先形成的化合物是磷锞 镨矿 $\left(\mathrm{LaPO}_{4} \cdot \mathrm{nH}_{2} \mathrm{O}\right)$. 磷镧镨矿的老化将使其逐渐转化为溶解性更低更稳定的独居石 $\left(\mathrm{LaPO}_{4}\right)^{[35]}$. 锞改性 膨润土对 SRP 有较强的吸附能力 ${ }^{[10,23]}$, 这在本研究中得到进一步验证, 加钵改性膨润土后上覆水体 SRP 大 幅下降. 而加锞改性膨润土后第 $1 \mathrm{~d}, \mathrm{TP}$ 和 PP 浓度却大幅上升, 因为在实际应用过程中会出现添加镧改性 膨润土后水体悬浮物浓度升高、透明度暂时性下降的现象 ${ }^{[36]}$, 锞改性膨润土本身所含有的磷以及 SRP 和 TDP 被吸附后尚未沉降可能是造成 TP 和 PP 浓度暂时升高的原因, 此外, 还可能是暨南大学南湖水深较浅, 镧改性膨润土投加迅速猛烈, 从而导致沉积物的再悬浮. 加镧改性膨润土后 (1 16 d), TP、PP、TDP 和 SRP 浓度均波动较大, 可能是大量降雨扰动所导致的, Bishop 等 ${ }^{[13]}$ 在 Laguna Niguel 湖的研究也出现了类似的结 果, 降雨导致加镧改性膨润土后上覆水体 TP 和 SRP 浓度仍然维持在较高的水平. 但 TP、PP、TDP 和 SRP 浓 度总体呈下降趋势, 后期 (33 60 d) 趋于平稳, 维持在较低的水平, 这表明镧改性膨润土改善水质的效果显 著,但具有一定的滞后性. 
镧改性膨润土沉降至沉积物后, 对沉积物活性磷具有较强的亲和力. 大量研究表明 ${ }^{[12,34]}$, 镧改性膨润土是 通过把活性磷 (主要包括 $\mathrm{NH}_{4} \mathrm{Cl}-\mathrm{P} 、 \mathrm{BD}-\mathrm{P}$ 和 Org-P) 转化为永久储存的惰性磷 (主要包括 HCl-P 和 Ref-P) 来 控制沉积物磷释放的. 固定在稀土镧中的磷 $\left(\mathrm{LaPO}_{4} \cdot \mathrm{nH}_{2} \mathrm{O}\right.$ 等) 大部分被 $\mathrm{HCl}$ 提取出来, 出现在 $\mathrm{HCl}-\mathrm{P}$ 中 $^{[9,34-35]}$. 本研究中加锞改性膨润土后 $60 \mathrm{~d}$, 南湖 $\mathrm{NH}_{4} \mathrm{Cl}-\mathrm{P}$ 含量在沉积物表层 $0 \sim 2 \mathrm{~cm}$ 范围显著下降, BD-P 含量在沉积物 $0 \sim 15 \mathrm{~cm}$ 范围内均显著下降, HCl-P 在沉积物表层 $0 \sim 6 \mathrm{~cm}$ 都有大幅度的增加, Ref-P 含量在 沉积物表层 2 $8 \mathrm{~cm}$ 范围显著增加, 说明镧改性膨润土在南湖中发挥了作用, 提高了沉积物对磷的滞留 能力.

沉积物活跃深度的估计值通常在 $4 \sim 10 \mathrm{~cm}^{[37]}$. Meis 等 ${ }^{[9]}$ 依据沉积物表层 $4 \mathrm{~cm}$ 中的活性磷含量在 Clatto 水库应用了镧改性膨润土, $28 \mathrm{~d}$ 后, 沉积物表层 $2 \mathrm{~cm}$ 的 Ref-P 含量显著增加, 表明锞改性膨润土提高了沉积 物对磷的束缚能力. 南湖镧改性膨润土的添加量更为保守, 是以沉积物表层 $5 \mathrm{~cm}$ 中的活性磷含量作为依 据, 添加量约为 $590 \mathrm{~g} / \mathrm{m}^{2}$, 稍高于厂家的推荐剂量 $\left(500 \mathrm{~g} / \mathrm{m}^{2}\right)$.

磷形态在沉积物较深层发生变化, 可能是因为 La 在垂直方向上发生了迁移. Meis 等 ${ }^{[9]}$ 研究发现, 在添 加镧改性膨润土 $28 \mathrm{~d}$ 后, 沉积物表层 $8 \mathrm{~cm}$ 中的镧含量显著增加, 我们在湖北省鄂州市洋澜湖的研究也发 现, 以 $500 \mathrm{~g} / \mathrm{m}^{2}$ 的剂量添加锞改性膨润土后 $70 \mathrm{~d}$, La 含量在沉积物表层 $0 \sim 10 \mathrm{~cm}$ 范围内显著增加（未发表 的数据).

沉积物 TP 含量在沉积物表层 0 $6 \mathrm{~cm}$ 稍有增加, 原因之一可能是水体中的磷被固定到了沉积物中, 另 外, 研究期间正值雨季, 地表径流形成的外源磷污染沉积到湖底, 可能也是导致表层 TP 增加原因之一. Org-P 含量变化不明显, 这可能是由于实验期间处于植物的生长旺季, 植物提供了 Org-P, 从而减弱了镧改性膨润 土对 Org-P 的作用,王华光等 ${ }^{[38]}$ 的研究具有相似的结果. Ref-P 在表层 $0 \sim 2 \mathrm{~cm}$ 变化不明显, 可能是因为该 层锞含量较高, 活性磷主要与镧形成了磷针镨矿, 而出现在了 HCl-P 中.

暨南大学南湖水深较浅, 处于亚热带, 雨水充足, 沉积物具有含水率高、有机质含量高、活性磷含量高等 特点,针改性膨润土在南湖的应用体现了其较好的环境条件适应性.

\section{4 结论}

1) 暨南大学南湖加镧改性膨润土后第 $1 \mathrm{~d}$, 上覆水 TP 和 PP 浓度却大幅上升. 加铺改性膨润土后 $60 \mathrm{~d}$ 与加之前 $2 \mathrm{~d}$ 相比, 上覆水体中 TP、PP、TDP 和 SRP 浓度分别下降了 $45.21 \% 、 43.91 \% 、 47.79 \%$ 和 $84.55 \%$, 对 水质的改善具有一定的促进作用,但具有一定的滞后性.

2) 镧改性膨润土对沉积物磷形态特征具有较大影响, 降低了暨南大学南湖沉积物中的 $\mathrm{NH}_{4} \mathrm{Cl}-\mathrm{P}$ 和 BD-P 含量, 而提高 HCl-P 和 Ref-P 含量, 从而提高沉积物对磷的滞留能力, 抑制沉积物磷的释放.

3) 对于类似暨南大学南湖 (水深较浅、处于亚热带、污染严重等) 的湖泊, 按照镧改性膨润土的质量与 沉积物表层 $5 \mathrm{~cm}$ 中 $\mathrm{P}_{\text {mobile }}\left(\mathrm{NH}_{4} \mathrm{Cl}-\mathrm{P} 、 \mathrm{BD}-\mathrm{P}\right.$ 和 Org-P 之和) 以及上覆水体 TP 之和的质量比为 $100: 1$ 的添加比 例, 可以取得较好的控磷效果, 该添加剂量具有较好的效果, 可以为该地区该类型湖泊的钝化修复提供添加 依据.

\section{5 参考文献}

[ 1 ] Downing JA. Limnology and oceanography: two estranged twins reuniting by global change. Inland Waters, 2014, 4(2): $215-232$.

[ 2 ] Jing L, Liu X, Bai S et al. Effects of sediment dredging on internal phosphorus: A comparative field study focused on iron and phosphorus forms in sediments. Ecological Engineering, 2015, 82(3) : 267-271.

[ 3 ] Jeppesen E, Søndergaard M, Jensen JP et al. Lake responses to reduced nutrient loading-an analysis of contemporary longterm data from 35 case studies. Freshwater Biology, 2005, 50(10) : 1747-1771.

[ 4 ] Nausch M, Nausch G, Lass HU et al. P input by upwelling in the eastern Gotland Basin( Baltic Sea) in summer and its effects on filamentous cyanobacteria Estuarine. Coastal and Shelf Science, 2009, 83(4) : 434-442.

[ 5 ] Huser BJ, Futter M, Lee JT et al. In-lake measures for phosphorus control: the most feasible and cost-effective solution for long-term management of water quality in urban lakes. Water Research, 2016, 97: 142-152. 
[ 6 ] Copetti D, Finsterle K, Marziali L et al. Eutrophication management in surface waters using lanthanum modified bentonite: A review. Water Research, 2016, 97: 162-174.

[ 7 ] Douglas RW, Rippey B. The random redistribution of sediment by wind in a lake. Limnology and Oceanography, 2000 , 45 (3) : 686-694.

[ 8 ] Douglas GB, Hamilton DP, Robb MS et al. Guiding principles for the development and application of solid-phase phosphorus adsorbents for freshwater ecosystems. Aquatic Ecology, 2016, 50(3) : 385-405.

[ 9 ] Meis S, Spears BM, Maberly SC et al. Sediment amendment with Phoslock in Clatto Reservoir ( Dundee, UK) : investigating changes in sediment elemental composition and phosphorus fractionation. Journal of Environmental Management, $2012,93(1): 185-193$.

[10] Spears BM, Lürling M, Yasseri S et al. Lake responses following lanthanum-modified bentonite clay ( Phoslock ${ }^{\mathrm{R}}$ ) application: An analysis of water column lanthanum data from 16 case study lakes. Water Research, 2013, 47( 15) : 5930-5942.

[11] Ross G, Haghseresht F, Cloete TE et al. The effect of pH and anoxia on the performance of Phoslock ( R), a phosphorus binding clay. Harmful Algae, 2008, 7(4) : 545-550.

[12] Meis S, Spears BM, Maberly SC et al. Assessing the mode of action of Phoslock ${ }^{\mathrm{R}}$ in the control of phosphorus release from the bed sediments in a shallow lake (Loch Flemington, UK). Water Research, 2013, 47(13) : 4460-4473.

[13] Bishop WM, Nabb TM, Cormican I et al. Hyde operational evaluation of Phoslock phosphorus locking technology in Laguna Niguel Lake, California. Water, Air, Soil and Pollution, 2014, 225: 2018.

[14] Zeng HY, Zhong P, Liu ZW et al. Response of metazoan zooplankton communities to ecological restoration in a tropical shallow lake. J Lake Sci, 2016, 28(1) : 170-177. DOI: 10.18307/2016.0120. [曾海逸, 钟萍, 刘正文等. 热带浅水湖 泊后生浮游动物群落结构对生态修复的响应. 湖泊科学, 2016, 28(1): 170-177.]

[15] Douglas GB. Remediation material and remediation process for sediments. US Patent 6350383, 2002.

[16] Editorial board of "Water and wastewater monitoring and analysis method", Ministry of Environmental Protection of the People's Republic of China ed. Monitoring and analysis methods of water and wastewater: fourth edition. Beijing: China Environmental Science Press, 2002. [国家环境保护总局《水和废水监测分析方法》编委会. 水和废水监测分析方 法: 第 4 版. 北京: 中国环境科学出版社, 2002.]

[17] Pettersson K, Boström B, Jacobsen OS et al. Phosphorus in sediments-speciation and analysis. Hydrobiologia, 1988, 170: 91-101.

[18] Ribeiro DC, Martins G, Nogueira R et al. Phosphorus fractionation in volcanic lake sediments (Azores-Portugal). Chemosphere, 2008, 70(7): 1256-1263.

[19] Krom MD, Berner RA. Adsorption of phosphate in anoxic marine sediments1. Limnology and Oceanography, 1980, 25 (5) : 797-806.

[20] Zhu GW, Gao G, Qin BQ et al. Geochemical characteristics of phosphorus in sediments of a large shallow lake. Advances in Water Science, 2003, 14(6): 714-719. [朱广伟, 高光, 秦伯强等. 浅水湖泊沉积物中磷的地球化学特征. 水科学进 展, $2003,14(6): 714-719$.

[21] Heiri O, Lotter A, Lemcke G. Loss on ignition as a method for estimating organic and carbonate content in sediments : Reproducibility and comparability of results. Journal of Paleolimnology, 2001, 25(1) : 101-110.

[22] Shen QS, Liu C, Zhou QL et al. Effects of physical and chemical characteristics of surface sediments in the formation of shallow lake algae-induced black bloom. Journal of Environmental Sciences, 2013, 25(12) : 2353-2360.

[23] Zhu GW, Li J, Zhu MY et al. Efficacy of Phoslock ${ }^{\mathrm{R}}$ on the reduction of sediment phosphorus release in West Lake, Hangzhou, China. Environmental Science, 2017, 38(4)：1451-1459. [朱广伟, 李静, 朱梦圆等. 锁磷剂对杭州西湖底泥 磷释放的控制效果. 环境科学, 2017, 38(4): 1451-1459.]

[24] Fan CX ed. Sediment-water interface of lakes, processes and effects. Beijing: Science Press, 2013. [范成新. 湖泊沉积 物界面过程与效应. 北京: 科学出版社, 2013.]

[25] Miao H, Shen Z, Jiang Y et al. Distribution characteristics and pollution assessment of nitrogen, phosphorus and organic matter in surface sediments of Chaohu Lake. Ecology and Environmental Sciences, 2017, 26(12) : 2120-2125. [苗慧, 沈 峥, 蒋豫等. 巢湖表层沉积物氮、磷、有机质的分布及污染评价，生态环境学报，2017，26(12): 2120-2125.]

[26] Yuan HZ, Shen J, Liu EF et al. Space distribution characteristics and diversity analysis of phosphorus from overlying water and surface sediments in Taihu Lake. Environmental Science, 2010, 31(4) : 954-960. [袁和忠, 沈吉, 刘恩峰等. 太湖 
水体及表层沉积物磷空间分布特征及差异性分析. 环境科学, 2010, 31(4): 954-960.]

[27] Zhao HC, Zhao HX, Wang SR et al. Effect of inorganic phosphorus forms in the sediment and soil by submerged plant. Ecology and Environment, 2008, 17(1): 74-80. [ 赵海超, 赵海香, 王圣瑞等. 沉水植物对沉积物及土壤垂向各形态 无机磷的影响. 生态环境, 2008, 17 (1) : 74-80.]

[28] Hupfer M, Dollan A. Immobilization of phosphorus by iron-coated roots of submerged macrophytes. Hydrobiologia, 2003, 506 $(1 / 2 / 3): 635-640$.

[29] Qin B, Xu P, Wu Q et al. Environmental issues of Lake Taihu, China. Hydrobiologia, 2007, 581 : 3-14.

[30] Zhang RY, Wu FC, Liu CQ et al. Characteristics of organic phosphorus fractions in different trophic sediments of lakes from the middle and lower reaches of Yangtze River region and Southwestern Plateau, China. Environmental Pollution, $2008, \mathbf{1 5 2}(2)$ : 366-372.

[31] James WF. Alum: redox-sensitive phosphorus ratio considerations and uncertainties in the estimation of alum dosage to control sediment phosphorus. Lake and Reservoir Management, 2005, 21(2) : 159-164.

[32] Rydin E, Huser B, Welch EB et al. Amount of phosphorus inactivated by alum treatments in Washington lakes. American Society of Limnology and Oceanography, 2000, 45(1): 226-230.

[33] Søndergaard M, Jensen JP, Jeppesen E. Role of sediment and internal loading of phosphorus in shallow lakes. Hydrobiologia, 2003, 506-509: 133-145.

[34] Reitzel K, Andersen FØ, Egemose S et al. Phosphate adsorption by lanthanum modified bentonite clay in fresh and brackish water. Water Research, 2013, 47( 8) : 2787-2796.

[35] Dithmer L, Lipton AS, Reitzel K et al. Characterization of phosphate sequestration by a lanthanum modified bentonite clay: a solid-state NMR, EXAFS and PXRD study. Environmental Science and Technology, 2015, 49(7) : 4559-4566.

[36] Waajen G, Oosterhout F, Douglasc G et al. Geo-engineering experiments in two urban ponds to control eutrophication. Water Research, 2016, 97: 69-82.

[37] Cooke GD, Welch EB, Peterson SA et al eds. Restoration and management of lakes and reservoirs: Third. London: Taylor \& Francis, 2005.

[38] Wang HG, Wang LY, Wang WJ et al. Effect of phoslock on the phosphorus forms in sediments with fluxion water condition. Environmental Pollution and Control, 2015, 37(7): 41-46. [王华光, 王凌燕, 王文静等. 锁磷剂对流动水环境 中沉积物磷形态的作用. 环境污染与防治, 2015, 37(7) : 41-46.] 